\title{
Clues to the past and inspiration for the future
}

\author{
by Gregory D. Larsen
}

\section{SCIENTIFIC NAME \\ Gekko gecko}

\section{TAXONOMY}

PHYLUM: Chordata

CLASS: Reptilia

ORDER: Squamata

FAMILY: Gekkonidae

\section{Physical description}

The Tokay gecko (Gekko gecko) is a nocturnal arboreal lizard whose native range spans the rainforests of south and southeast Asia, from India through New Guinea. Tokay geckos are one of the largest gecko species, growing up to $51 \mathrm{~cm}$ in length and weighing up to $400 \mathrm{~g}$. Their skin is generally gray with reddish spots and can lighten or darken in coloration to blend in with surroundings. Tokay geckos have large eyes with a vertically slit pupil and a dorsally located pineal eye.

Tokay geckos have a distinctive vocal mating call, from which both names, 'Tokay' and 'gecko', are onomatopoeically derived. Males are highly territorial and, outside of mating, these geckos are solitary animals. Like many gecko species, Tokay geckos feed on insects and small invertebrates, which they hunt from nearly any angle and surface that their toes can grip.

\section{Research résumé}

Many studies use geckos to explore questions of comparative physiology and evolutionary biology. Geckos are distant evolutionary relatives of birds and mammals, but as fellow amniotes, they are more similar to these groups than are other common models, such as salamanders and zebrafish. They are generally considered convenient to study because they are relatively hardy, can be commercially bred and have well-established husbandry protocols ${ }^{1}$.

As vocal communicators and visual hunters, geckos possess keen auditory and visual systems that distinguish them among reptiles. The Tokay gecko's auditory fibers possess tonal sensitivity and the capacity to 'tune in' to selective frequencies ${ }^{2}$, while their eyes possess differentiated, rod-like photoreceptors that enhance their nocturnal vision $^{3}$. These traits are common and specialized among different gecko species, though rare among other lizards, and their various forms might hold clues as to how these complex senses evolved in other lineages of terrestrial vertebrates.

Like many reptiles and amphibians, geckos can sever their own tails and grow a similar replacement. Although the new tail is not a complete replica, it reflects regenerative capabilities far superior to those of most non-reptilian amniotes ${ }^{1}$. Many researchers have therefore investigated the mechanisms of the underlying process, hoping that they might advance regenerative medicine in humans.

Geckos are perhaps best known for their ability to scale and grip nearly any surface, which has inspired many studies and inventions in recent years. Many gecko species possess adhesive pads on their toes, which are covered in keratinous setae made up of hundreds of 200-nm spatulae ${ }^{4}$. As a gecko walks along a surface, these spatulae flatten against the substrate material and form weak 을 chemical bonds through vo van der Waals' interactions. The cumulative total adhesion of the bonds between spatulae and substrate is more than enough to support the gecko's body weight ${ }^{5}$. The strength, endurance and versatility of this adhesive effect surpass conventional manufactured adhesives and have inspired new biomimetic materials ${ }^{5,6}$ and even robots, one of which was recently presented at the 2015 International Conference on Robotics and Automation?

1. McLean, K.E. \& Vickaryous, M.K. A novel amniote model of epimorphic regeneration: the leopard gecko, Eublepharis macularius. BMC Dev. Biol. doi:10.1186/1471-213X-11-50 (published online 16 August 2011).

2. Sams-Dodd, F. \& Capranica, R.R. Representation of acoustic signals in the eighth nerve of the Tokay gecko: I. Pure tones. Hearing Res. 76, 16-30 (1994).

3. Röll, B. Gecko vision-visual cells, evolution, and ecological constraints. J. Neurocytol. 29, 471-484 (2000).

4. Hansen, W.R. \& Autumn, K. Evidence for self-cleaning in gecko setae. Proc. Natl. Acad. Sci. USA 102, 385-389 (2005).

5. Tian, Y. et al. Adhesion and friction in gecko toe attachment and detachment. Proc. Natl. Acad. Sci. USA 103, 19320-19325 (2006).

6. Zhou, M., Pesika, N., Zeng, H., Tian, Y. \& Israelachvili, J. Recent advances in gecko adhesion and friction mechanisms and development of gecko-inspired dry adhesive surfaces. Friction 1, 114-129 (2013).

7. Christensen, D.L. et al. $\mu$ Tugs: Enabling Microrobots to Deliver Macro Forces with Controllable Adhesives (IEEE International Conference on Robotics and Automation; Seattle, WA; 26-30 May 2015). 